\title{
Random Aspects of High-dimensional Convex Bodies
}

\author{
A.E. Litvak* and N. Tomczak-Jaegermann** \\ Department of Mathematical Sciences, University of Alberta, Edmonton, Alberta, \\ Canada T6G 2G1
}

\section{Introduction}

In this paper we study geometry of compact, not necessarily centrally symmetric, convex bodies in $\mathbb{R}^{n}$. Over the years, local theory of Banach spaces developed many sophisticated methods to study centrally symmetric convex bodies; and already some time ago it became clear that many results, if valid for arbitrary convex bodies, may be of interest in other areas of mathematics.

In recent years many results on non-centrally symmetric convex bodies were proved and a number of papers have been written (see e.g., [1], [8], [12], [18], [27], [28] among others). The present paper concentrates on random aspects of compact convex bodies and investigates some invariants fundamental in the local theory of Banach spaces, restricted to random sections and projections of such bodies. It turns out that, loosely speaking, such random operations kill the effect of non-symmetry in the sense that resulting estimates are very close to their centrally symmetric counterparts (this is despite the fact that random sections might be still far from being symmetric (see Section 5 below)). At the same time these estimates might be in a very essential way better than for general bodies.

We are mostly interested in two directions. One is connected with so-called $M M^{*}$ - estimate, and related inequalities. For a centrally symmetric convex body $K \subset \mathbb{R}^{n}$, an estimate $M(K) M\left(K^{0}\right) \leq c \log n$ (see the definitions in Section 2 below) is an important technical tool intimately related to the $K$ convexity constant. It follows by combining works by Lewis and by Figiel and Tomczak-Jaegermann, with deep results of Pisier on Rademacher projections (see e.g., [26]). Although the symmetry can be easily removed from the first two parts, Pisier's argument use it in a very essential way. In Section 4 we show, in particular, that every convex body $K$ has a position $K_{1}$ (i.e., $K_{1}=$ $u K-a$ for some operator $u$ and $a \in \mathbb{R}^{n}$ ) such that a random projection, $P K_{1}$, of dimension $[n / 2]$ satisfies $M\left(P K_{1}\right) M\left(K_{1}^{0}\right) \leq C \log n$, where $C$ is an absolute constant. Moreover, there exists a unitary operator $u$ such that $M\left(K_{1}+u K_{1}\right) M\left(K_{1}^{0}\right) \leq C \log n$. Our proof is based essentially on symmetric considerations, a non-symmetric part is reduced to classical facts and simple

\footnotetext{
* Current address: Department of Mathematics, Technion, Haifa, Israel 32000

** This author held the Canada Council Killam Research Fellowship in 1997/99.
} 
lemmas. Similar estimates were recently proved by a different method by Rudelson ([28]).

In Section 3 we develop some tools. We introduce the concept of random Gelfand numbers, which formalises the phenomenon of lower estimates by the Euclidean norm on random subspaces. Theorem 3.2 shows that, roughly speaking, for any convex body $K \subset \mathbb{R}^{n}$, good lower estimates on (nonrandom) subspaces of proportional dimensions imply similar estimates valid on random subspaces of comparable dimensions. The results of this section are new even for centrally symmetric bodies.

Our second source of invariants is related to distances between convex bodies. For symmetric convex bodies this theory has been much studied (see [30] and references therein); in contrast, for non-symmetric convex bodies very little is known in this direction (see [28] for a few recent results). In Section 5 we consider, for example, a natural way to measure a "non-symmetry" of a convex body, as the distance of a body to the set of all centrally symmetric bodies, and we investigate the behaviour of this distance for random sections (and projections) of convex bodies. It turns out that for random projections of rank $k$ of a simplex $S \subset \mathbb{R}^{n}$, this distance can be asymptotically estimated from below by $\sqrt{k / \ln n}$ (Theorem 5.1). On the other hand, the case of simplex is the worst (up to a logarithmic factor). This follows from Theorem 5.3 related to an "isomorphic" version of Dvoretzky's theorem ([20], [21]).

Finally, in Section 6 we discuss the so-called proportional DvoretzkyRogers factorization in the non-symmetric setting. The proof essentially follows the known symmetric argument ([29]), but it has a few delicate points and it needs some extra work (based on [5]). An interesting standard application says that every convex body has $k$-dimensional projection and section $(k=[n / 2])$ whose distances to $\ell_{1}^{k}$ and to $\ell_{\infty}^{k}$, respectively, are smaller than $C \sqrt{n}$ (where $C$ is an absolute constant). Another application, Theorem 6.7, extends the proofs of "isomorphic" version of Dvoretzky's theorem by Milman and Schechtman ([20], [21]) to the non-symmetric case (cf. [8]).

\section{Definitions and Notation}

Let $n$ be a positive integer. Denote the canonical Euclidean norm on $\mathbb{R}^{n}$ by $|\cdot|$ and the Euclidean unit ball by $D$. Given a set $A \subset \mathbb{R}^{n}$, denote the volume of $A$ by $|A|$. Given a subspace $E \subset \mathbb{R}^{n}$, denote the orthogonal projection on $E$ by $P_{E}$.

By a convex body $K \subset \mathbb{R}^{n}$ we shall always mean a compact convex set with a non-empty interior, and without loss of generality, we shall assume that $0 \in \operatorname{Int} K$. The gauge of $K$ denoted by $\|\cdot\|_{K}$ is a positive convex homogeneous functional; and $X=\left(\mathbb{R}^{n},\|\cdot\|_{K}\right)$ is an $n$-dimensional linear space, corresponding to the functional. This space will be also denoted by $\left(\mathbb{R}^{n}, K\right)$. For the Banach space notation which corresponds to the case of 
centrally symmetric convex bodies we refer the reader to [19], [26] and [30]. In particular, an operator always means a linear operator.

For an arbitrary $z \in \operatorname{Int} K$, by $K^{z}$ we denote the polar body with respect to $z$, that is, $K^{z}=\{y+z \mid(y, x-z) \leq 1$ for all $x \in K\}$. So, $K^{0}$ is the standard polar of $K$. Recall that for every convex body $K$ there is a unique point $z \in \operatorname{Int} K$, called a Santaló point, such that $|K|\left|K^{z}\right| \leq|D|^{2}$. We shall say that $z \in \operatorname{Int} K$ is an isomorphic Santaló's point for $K$ (with constant $C$ ), if $|K|\left|K^{z}\right| \leq C^{n}|D|^{2}$.

Let us recall the notation connected with distances.

Given convex bodies $K, L$ in $\mathbb{R}^{n}$, we define the geometric distance by

$$
\widetilde{d}(K, L)=\inf \{\alpha \beta \mid(1 / \beta) L \subset K \subset \alpha L\} .
$$

The Banach-Mazur distance is defined by

$d(K, L)=\inf \left\{\widetilde{d}(u(K-z), L-x) \mid x, z \in \mathbb{R}^{n} ; u: \mathbb{R}^{n} \rightarrow \mathbb{R}^{n}\right.$ an isomorphism $\}$

and it corresponds to the notion of the Banach-Mazur distance in the centrally symmetric case, i.e. if $K$ and $L$ are centrally symmetric convex bodies then to attain the infimum in the definition it is enough to take $z=x=0$. Given convex body $K$ in $\mathbb{R}^{n}$ and centrally symmetric convex body $L$ in $\mathbb{R}^{n}$ we define the distance corresponding to a center $z$ by

$$
d_{z}(K, L)=\inf \left\{\widetilde{d}(u(K-z), L) \mid u: \mathbb{R}^{n} \rightarrow \mathbb{R}^{n} \text { an isomorphism }\right\} .
$$

Clearly, $d(K, L) \leq 2 \inf \left\{d_{z}(K, L) \mid z \in \mathbb{R}^{n}\right\}$. For simplicity below we will use $d(K, L)$ for the $\inf \left\{d_{z}(K, L) \mid z \in \mathbb{R}^{n}\right\}$ in the case $L=-L$.

For a convex body $K \subset \mathbb{R}^{n}$ we use shorthand notation $d_{K}=d(K, D)$ and $d_{K, z}=d_{z}(K, D)$ for $z \in \mathbb{R}^{n}$.

Many well known definitions for centrally symmetric bodies are naturally extended to the non-symmetric case by the same formulas. Let $K \subset \mathbb{R}^{n}$ be a convex body. Recall that

$$
M=M_{K}=M(K)=\int_{S^{n-1}}\|x\|_{K} d \nu(x),
$$

where $S_{n-1}$ denotes the Euclidean unit sphere and $\nu$ the normalized Haar measure on $S_{n-1}$. Let $M^{*}=M_{K}^{*}=M^{*}(K)=M\left(K^{0}\right)$. It is easy to check that

$$
M(K) \leq M(K \cap-K) \leq 2 M(K) .
$$

Let

$$
\ell(K)=\mathbf{E}\left\|\sum_{i=1}^{n} g_{i} e_{i}\right\|_{K},
$$

where $g_{i}$ are independent standard Gaussian random variables, and $\left\{e_{i}\right\}$ is the canonical basis of $\mathbb{R}^{n}$. It is well known that $\ell(K)=c_{n} M(K) \sqrt{n}$, where $c_{n}<1$ and $c_{n} \rightarrow 1$ as $n \longrightarrow \infty$. 
For a centrally symmetric convex body $B \subset \mathbb{R}^{n}$ the $K$-convexity constant of $\left(\mathbb{R}^{n}, B\right)$ is denoted by $\kappa(B)$. It is well known that there exists an isomorphism $u: \mathbb{R}^{n} \longrightarrow \mathbb{R}^{n}$ such that the body $\widetilde{B}=u(B)$ satisfies $M(\widetilde{B}) M\left(\widetilde{B}^{0}\right) \leq C \kappa(B) \leq C^{\prime} \log d_{B}$, where $C$ and $C^{\prime}$ are absolute constants. ( $u$ is determined by the so-called $\ell$-ellipsoid for $B$, and the latter estimate is the well-known estimate by Pisier, cf. e.g., [19], [26], [30].)

For $1 \leq k \leq n$, by $\mu_{n, k}$ we denote the normalized Haar measure on the Grassmann manifold $G_{n, k}$ of all $k$-dimensional subspaces of $\mathbb{R}^{n}$.

Let us recall the so-called "lower $M^{*}$-estimate".

Theorem 2.1 Let $K \subset \mathbb{R}^{n}$ be a convex body. For every $1 \leq k \leq n$, the set of all subspaces $E \subset \mathbb{R}^{n}$ with codim $E=k-1$ such that

$$
\sqrt{k / n}|x| \leq 2 M^{*}(K)\|x\|_{K} \quad \text { for all } x \in E,
$$

has measure larger than $1-\exp \left(-\alpha_{0} k\right)$, where $\alpha_{0}>0$ is an absolute constant.

The first estimate of this type was proved by Milman ([14]) with a certain function $f(\lambda)$ replacing $\sqrt{\lambda}$ on the left hand side, where $\lambda=k / n \in(0,1)$, and the factor 2 replaced by an absolute constant. Then $f$ was improved to a polynomial by Milman ([15], [16]), and to the present form (which is asymptotically optimal) by Pajor and Tomczak-Jaegermann ([23]). Subsequently, Gordon ([7]) improved the factor on the right hand side (see also [17]). The non-symmetric case has been known for a long time and is obtained by all the methods above.

Let us finally mention that following the practise typical for the local theory of Banach spaces, we write most of our results in terms of convex bodies rather than operators acting between two such bodies. Passing to the operator language is completely standard.

\section{Random Gelfand Numbers}

The concept of random subspaces is fundamental in large parts of the theory. In this paper, given a property of $m$-dimensional subspaces of $\mathbb{R}^{n}$, we say that this property is satisfied by a random $m$-dimensional subspace if the measure $\mu_{n, m}$ of the subset of $G_{n, m}$ of all subspaces satisfying this property is larger than $1-\exp \left(-\alpha_{0}(n-m+1)\right)$.

For sake of generality, in the next definitions we consider arbitrary operators. Let $K, L \subset \mathbb{R}^{n}$ be convex bodies and let $u:\left(\mathbb{R}^{n}, K\right) \longrightarrow\left(\mathbb{R}^{n}, L\right)$ be an operator. Let $1 \leq k \leq n$. First we recall a classical definition of Gelfand numbers, $c_{k}(u)$. Let

$$
c_{k}(u)=\inf \left\{\max _{x \in E}\|u x\|_{L} /\|x\|_{K} \mid E \subset \mathbb{R}^{n}, \operatorname{codim} E<k\right\} .
$$


Definition 3.1 We define the $k$-th random Gelfand number by $c r_{k}(u)=$ $\inf a$, where $a>0$ is a real number such that the inequality

$$
a>\max _{x \in E}\|u x\|_{L} /\|x\|_{K}
$$

is satisfied for a random subspace $E$ of $\operatorname{codim} E=k-1$.

We shall write $c_{k}(K)$ and $c r_{k}(K)$ to denote $c_{k}(i d)$ and $c r_{k}(i d)$, respectively, where $i d:\left(\mathbb{R}^{n}, K\right) \longrightarrow\left(\mathbb{R}^{n}, D\right)$ is the formal identity operator.

Theorem 2.1 can then be reformulated as follows.

Theorem 2.1'. Let $K \subset \mathbb{R}^{n}$ be a convex body. For every $1 \leq k \leq n$ we have

$$
\sqrt{k} c r_{k}(K) \leq 2 \ell\left(K^{0}\right) .
$$

Let us recall a definition well-known in the symmetric case. For a convex body $K \subset \mathbb{R}^{n}$, and $1 \leq k \leq n$, the volume number $v_{k}(K)$ is defined by

$v_{k}(K)=\sup \left\{(|P K| /|P D|)^{1 / k} \mid P\right.$ is an orthogonal projection of rank $\left.k\right\}$.

Theorem 3.2 Let $K \subset \mathbb{R}^{n}$ be a convex body. For every $1 \leq k \leq n$ we have

$$
\sqrt{k} c r_{k}(K) \leq C \sum_{j=m}^{n} c_{j}(K) / \sqrt{j},
$$

where $m=[c k]$, and $c>0$ and $C>1$ are absolute constants. Moreover, if 0 is an isomorphic Santaló point for $K$ (with constant $C^{\prime}$ ), then we also have

$$
\sum_{j=m}^{n} c_{j}(K) / \sqrt{j} \leq C^{\prime \prime}(\lambda) \sum_{j=[m / 2]}^{n} v_{j}(K) / \sqrt{j}
$$

where $\lambda=k / n$ and $C^{\prime \prime}(\lambda)$ depends on $C^{\prime}$ and $\lambda$.

Proof. The moreover part in the symmetric case is (1.13) of [24]. Since this inequality follows from Milman's quotient-subspace theorem (see the proof in [13]), it holds in the non-symmetric case, as long as the choice of the center ensures the validity of Milman's theorem. This in turn was shown in [18] and [28] to depend on the inequality (7) below to be satisfied for $K$. Finally, if 0 is an isomorphic Santaló point for $K$, then (7) holds for $K$.

The first part requires an additional notation. For a convex body $K \subset \mathbb{R}^{n}$ and $\rho>0$, set $K_{\rho}=K \cap \rho D$, and by $\|x\|_{\rho}$ denote the gauge

$$
\|x\|_{K_{\rho}}=\max \left\{\|x\|_{K},|x| / \rho\right\} .
$$

The following lemma is analogous to (4) of [22]. 
Lemma 3.3 Let $K \subset \mathbb{R}^{n}$ be a convex body and let $\rho=\inf \beta$, over all $\beta$ such that

$$
\mu_{n, n-k+1}\left(\left\{E \mid \operatorname{codim} E=k-1, \beta>\max _{x \in E} \frac{|x|}{\|x\|_{\beta}}\right\}\right) \geq 1-\exp \left(-\alpha_{0} k\right) .
$$

Then

$$
\rho=c r_{k}\left(K_{\rho}\right)=c r_{k}(K) \text {. }
$$

Proof. Clearly, $\operatorname{cr}_{k}\left(K_{\rho}\right) \leq c r_{k}(K)$. Write $\|\cdot\|$ for $\|\cdot\|_{K}$, and $\mu$ for $\mu_{n, n-k+1}$, and let $\alpha_{k}=1-\exp \left(-\alpha_{0} k\right)$.

Given $\beta$ denote

$$
A_{\beta}:=\left\{E \mid \operatorname{codim} E=k-1, \beta>\max _{x \in E} \frac{|x|}{\|x\|_{\beta}}\right\}
$$

and

$$
A_{\beta}^{\prime}:=\left\{E \mid \operatorname{codim} E=k-1, \beta>\max _{x \in E} \frac{|x|}{\|x\|}\right\} .
$$

Since $A_{\beta}^{\prime} \subset A_{\beta}$, we have $\mu\left(A_{\beta}^{\prime}\right) \leq \mu\left(A_{\beta}\right)$; and, by definition, for every $\beta>\rho$ if $E \in A_{\beta}$ then $\|x\|_{\beta}=\|x\|$ for every $x \in E$. Therefore $\mu\left(A_{\beta}^{\prime}\right)=\mu\left(A_{\beta}\right)$. Thus,

$$
c r_{k}(K)=\inf \left\{\beta \mid \mu\left(A_{\beta}^{\prime}\right) \geq \alpha_{k}\right\} \leq \inf \left\{\beta>\rho \mid \mu\left(A_{\beta}^{\prime}\right) \geq \alpha_{k}\right\}=\rho .
$$

On the other hand for every $\beta<\rho$ one has

$$
\mu\left(\left\{E \mid \operatorname{codim} E=k-1, \beta>\max _{x \in E} \frac{|x|}{\|x\|_{\rho}}\right\}\right) \leq \mu\left(A_{\beta}\right)<\alpha_{k},
$$

which means $\operatorname{cr}_{k}\left(K_{\rho}\right) \geq \rho$.

Let us also recall the result based on the Dudley theorem (see e.g., [26], Chapter 5; one can easily check that the argument works in the nonsymmetric case as well). It says that if $K \subset \mathbb{R}^{n}$ is a convex body then

$$
\ell\left(K^{0}\right) \leq C_{0} \sum_{j=1}^{n} c_{j}(K) / \sqrt{j}
$$

where $C_{0}>1$ is an absolute constant.

Returning to the proof of Theorem 3.2, let $\rho$ be as in the lemma. Then by Theorem $2.1^{\prime}$ and (2) one has

$$
\rho=c r_{k}(K)=c r_{k}\left(K_{\rho}\right) \leq \frac{2 C_{0}}{\sqrt{k}} \sum_{j=1}^{n} c_{j}\left(K_{\rho}\right) / \sqrt{j}
$$


Since for any $j$

$$
c_{j}\left(K_{\rho}\right) \leq\left\|i d:\left(\mathbb{R}^{n}, K_{\rho}\right) \longrightarrow\left(\mathbb{R}^{n}, D\right)\right\|=\rho,
$$

we have for every $m$

$$
\begin{aligned}
\rho & \leq \frac{2 C_{0}}{\sqrt{k}}\left(\sum_{j=1}^{m} \rho / \sqrt{j}+\sum_{j=m+1}^{n} c_{k}\left(K_{\rho}\right) / \sqrt{j}\right) \\
& \leq \frac{4 C_{0}}{\sqrt{k}} \sqrt{m} \rho+\frac{2 C_{0}}{\sqrt{k}} \sum_{j=m+1}^{n} c_{k}(K) / \sqrt{j} .
\end{aligned}
$$

Choosing $m=\left[k /\left(12 C_{0}\right)^{2}\right]$ we complete the proof of the theorem, for appropriate constants $c$ and $C$.

Remark 3.4 It is well known that in centrally symmetric case $C^{\prime \prime}(\lambda)$ in Theorem 3.2 can be taken as $c /(\lambda \ln \lambda)$, where $c$ is an absolute constant. In the general case that function can be taken as $c /\left(\lambda \ln ^{a} \lambda\right)$, where $c$ and $a$ are absolute constants ([28]).

Theorem 3.5 Let $K \subset \mathbb{R}^{n}$ be a convex body such that 0 is an isomorphic Santaló point for $K$ (with constant $C^{\prime}$ ) and let $1 \leq k \leq n$. For a random subspace $E$ with codim $E=k-1$ one has

$$
\ell\left(P_{E} K^{0}\right) \leq C^{\prime}(\lambda) \sum_{j=[c k]}^{n} v_{j}(K) / \sqrt{j}
$$

where $c>0$ is an absolute constant, $\lambda=k / n$ and $C^{\prime}(\lambda)>1$ depends on $C$ and $\lambda$.

Proof. Let $\rho$ be as in Lemma 3.3 and $\beta \leq 2 \rho$ such that $\mu_{n, n-k+1}\left(A_{\beta}\right) \geq \alpha_{k}$, where $A_{\beta}$ and $\alpha_{k}$ were defined in the proof of Lemma 3.3. Then

$$
\rho=c r_{k}\left(K_{\rho}\right) \leq c r_{k}\left(K_{\beta}\right) \leq c r_{k}(K)=\rho .
$$

Repeating the argument of Theorem 3.2 and setting $K_{\beta}^{0}=\left(K_{\beta}\right)^{0}$, we get

$$
\begin{aligned}
\ell\left(K_{\beta}^{0}\right) & \leq C_{0} \sum_{j=1}^{n} c_{j}\left(K_{\beta}\right) / \sqrt{j} \leq \frac{4 C_{0}}{\sqrt{k}} \sum_{j=m}^{n} c_{j}(K) / \sqrt{j} \\
& \leq C(\lambda) \sum_{j=m / 2}^{n} v_{k}(K) / \sqrt{j}
\end{aligned}
$$

where $m=\left[k /\left(24 C_{0}\right)^{2}\right]($ since $\beta \leq 2 \rho)$. 
Moreover, for every $E \in A_{\beta}$ we have

$$
\left(P_{E} K_{\beta}^{0}\right)_{E}^{0}=K_{\beta} \cap E=K \cap E,
$$

where $\left(P_{E} K_{\beta}^{0}\right)_{E}^{0}$ denotes polar to $P_{E} K_{\beta}^{0}$ with respect to the subspace $E$. This means that

$$
P_{E} K_{\beta}^{0}=P_{E} K^{0} .
$$

Thus, by the ideal property of the $\ell$-functional we get

$$
\ell\left(P_{E} K^{0}\right)=\ell\left(P_{E} K_{\beta}^{0}\right) \leq \ell\left(K_{\beta}^{0}\right)
$$

which completes the proof.

\section{$4 \quad M M^{*}$ Estimates for Convex Bodies}

For a non-symmetric convex body the usual meaning of a position (that is, an image of the body by a linear operator) has to be modified to reflect the importance of the choice of the center.

Given a convex body $K \subset \mathbb{R}^{n}$, we say that a body $K_{1}$ is a position of $K$ if for some absolute constant $C$ we have:

1. $K_{1}=u K-a$ for some isomorphism $u$ and some $a \in \mathbb{R}^{n}$,

2. $d_{0}\left(K_{1}, D\right) \leq C d_{K}$,

3. $\left|K_{1}\right| \cdot\left|K_{1}^{0}\right| \leq C^{n}|D|^{2}$.

From Lemmas 4.4 and 4.5 below it follows that the set of $a \in \mathbb{R}^{n}$ satisfying conditions 2 and 3 is non-empty.

The main result in this section is the following theorem.

Theorem 4.1 Let $K \subset \mathbb{R}^{n}$ be a convex body. There exists a position $K_{1}$ of $K$ such that for every $0<\lambda<1$, a random subspace $E \subset \mathbb{R}^{n}$ with $\operatorname{dim} E=[\lambda n]$ satisfies

$$
M\left(P_{E}\left(K_{1} \cap-K_{1}\right)\right) M\left(K_{1}^{0}\right) \leq C(\lambda) \kappa(K-K) \leq C^{\prime}(\lambda) \log \left(1+d_{K}\right),
$$

where $C(\lambda)$ and $C^{\prime}(\lambda)$ depend on $\lambda$ only.

This theorem can be reformulated in a global form.

Theorem 4.1'. Let $K \subset \mathbb{R}^{n}$ be a convex body. There exists a position $K_{1}$ of $K$ such that, letting $B=K_{1} \cap-K_{1}$, there exists a unitary operator $U$ satisfying

$$
M(B+U B) M\left(K_{1}^{0}\right) \leq C \kappa(K-K),
$$

where $C>1$ is an absolute constant. 
Proof. Let $E \subset \mathbb{R}^{n}$ be a random [n/2]-dimensional subspace such that the both projections $P=P_{E}$ and $P^{\prime}=P_{E^{\perp}}$ satisfy the conclusion of Theorem 4.1. Set $U=P-P^{\prime}$. Since $B$ is a symmetric body, we clearly have $P B+P^{\prime} B \subset B+U B$; and so $M(B+U B) \leq M\left(P B+P^{\prime} B\right)$. Since $P B$ and $P^{\prime} B$ are contained in mutually orthogonal subspaces of $\mathbb{R}^{n}$, the latter quantity is less than or equal to $2\left(M(P B)+M\left(P^{\prime} B\right)\right)$. The conclusion follows by the choice of $E$.

The above proof is modeled on an argument from [12].

Before going on, let us discuss several consequences of Theorem 4.1. The first one means that if we pass to random subspace $E$ of $\mathbb{R}^{n}$ then the body $\widetilde{K}=P_{E} K$ satisfies the same estimate for $M M^{*}$ as in the symmetric case. (This is despite the fact that $\widetilde{K}$ may happen to be quite far from being symmetric.)

Corollary 4.2 Let $K \subset \mathbb{R}^{n}$ be a convex body. There exists a position $K_{1}$ of $K$ such that for every $0<\lambda<1$, a random subspace $E \subset \mathbb{R}^{n}$ with $\operatorname{dim} E=[\lambda n]$ satisfies

$$
M\left(P_{E} K_{1}\right) M\left(K_{1}^{0} \cap E\right) \leq C(\lambda) \kappa(K-K) \leq C^{\prime}(\lambda) \log \left(1+d_{K}\right),
$$

where $C(\lambda)$ and $C^{\prime}(\lambda)$ depend on $\lambda$ only.

The second corollary allows to pass from a projection of a body to a section, but in doing so we lose randomness.

Corollary 4.3 Let $K \subset \mathbb{R}^{n}$ be a convex body. There exists a position $K_{1}$ of $K$ such that for every $0<\lambda<1$, there exists a subspace $E \subset \mathbb{R}^{n}$ with $\operatorname{dim} E=[\lambda n]$ such that

$$
M\left(K_{1} \cap E\right) M\left(K_{1}^{0}\right) \leq C(\lambda) \kappa(K-K) \kappa\left(K_{1} \cap-K_{1}\right) \leq C^{\prime}(\lambda) \log ^{2}\left(1+d_{K}\right),
$$

where $C(\lambda)$ and $C^{\prime}(\lambda)$ depend on $\lambda$ only.

This easily follows from Theorem 4.1, by using the lifting property of the $\ell$-functional (see Lemma 9.4 of [26] and the remark afterwards).

Corollary 4.3 was proved by a different method by Rudelson ([28], see also [27], for the main technical step), who obtained a better dependence of $C(\lambda)$ on $\lambda$.

We now start the proof of Theorem 4.1, which requires some preparation.

Lemma 4.4 Let $K \subset \mathbb{R}^{n}$ be a convex body (with $0 \in \operatorname{Int} K$ ). Let $z \in(1 / 2) K$. Then the polar $K^{z}$ with respect to $z$ satisfies $\left|K^{z}\right| \leq 2^{n}\left|K^{0}\right|$. 
Proof. By definition

$$
K^{z}-z=\{y \mid(y, x-z) \leq 1 \text { for all } x \in K\} .
$$

However if $(y, x) \leq 1+(y, z)$ then $\|y\|_{K^{0}} \leq 1+(1 / 2)\|y\|_{K^{0}}$, because $z \in$ $(1 / 2) K$. That means $K^{z}-z \subset 2 K^{0}$ which implies the result.

Lemma 4.5 Let $K \subset \mathbb{R}^{n}$ be a convex body (with $0 \in \operatorname{Int} K$ ). Let $a \in K$ and let $0<\theta<1$. Then

$$
d_{K, \theta a} \leq\left(\frac{2}{\theta}-1\right) d_{K, a} .
$$

Proof. Denote $d_{K, a}$ by $d$. Let $\mathcal{E}$ be an ellipsoid centered at 0 such that $a+\mathcal{E}$ $\subset K \subset a+d \mathcal{E}$. Clearly $\theta a+\theta \mathcal{E} \subset \theta K \subset K$. On the other hand, since $0 \in K \subset a+d \mathcal{E}$ then $-a \in d \mathcal{E}$, hence $a \in d \mathcal{E}$. Thus $K \subset \theta a+(2-\theta) d \mathcal{E}$. Therefore, $d_{K, \theta a} \leq((2-\theta) / \theta) d$.

The key ingredient of our approach is contained in the following proposition for symmetric convex bodies.

Proposition 4.6 Let $B_{1} \subset B_{2} \subset \mathbb{R}^{n}$ be symmetric convex bodies. For every $0<\lambda<1$, a random subspace $E \subset \mathbb{R}^{n}$ with $\operatorname{dim} E=[\lambda n]$ satisfies

$$
\ell\left(P_{E} B_{1}\right) \leq C(\lambda)\left(\frac{\left|B_{2}\right|}{\left|B_{1}\right|}\right)^{c / k} \ell\left(B_{2}\right),
$$

where $k=n-[\lambda n], C(\lambda)$ depend on $\lambda$ only, and $c$ is an absolute constant.

The proof requires the definition of covering and entropy numbers. Recall that for arbitrary subsets $K_{1}, K_{2}$ of $\mathbb{R}^{n}$ the covering number $N\left(K_{1}, K_{2}\right)$ is defined as the smallest number $N$ such that there exist $N$ points $y_{1}, \ldots, y_{N}$ in $\mathbb{R}^{n}$ satisfying

$$
K_{1} \subset \bigcup_{i=1}^{N}\left(y_{i}+K_{2}\right) .
$$

The entropy numbers are defined by

$$
e_{k}\left(K_{1}, K_{2}\right)=\inf \left\{\varepsilon>0 \mid N\left(K_{1}, \varepsilon K_{2}\right) \leq 2^{k-1}\right\} .
$$

It is known (and easy to check) that for an arbitrary convex body $K \subset \mathbb{R}^{n}$ one has $v_{k}(K) \leq 2 e_{k}(K, D)$ (see e.g., [26], ch. 9).

Proof of Proposition 4.6. By Theorem 3.5 for a random subspace $E \subset \mathbb{R}^{n}$ with codim $E=k=n-[\lambda n]$ one has

$$
\ell\left(P_{E} B_{1}\right) \leq C(\lambda) \sum_{m=[c k]}^{n} \frac{v_{m}\left(B_{1}^{0}\right)}{\sqrt{m}}
$$


where $c$ is numerical constants and $v_{m}\left(B_{1}^{0}\right)$ are the volume numbers of $B_{1}^{0}$. Let $\varepsilon=e_{n}\left(B_{2}^{0}, D\right)$ (entropy number). Since $B_{2}^{0} \subset B_{1}^{0}$ and by definition of entropy numbers we have

$$
N:=N\left(B_{1}^{0}, \varepsilon D\right) \leq N\left(B_{1}^{0}, B_{2}^{0}\right) N\left(B_{2}^{0}, \varepsilon D\right) \leq 3^{n} \frac{\left|B_{1}^{0}\right|}{\left|B_{2}^{0}\right|} 2^{n}
$$

(the latter inequality follows from Lemma 4.16 of [26] and the choice of $\varepsilon$ ). Therefore there are $N$ points $x_{1}, \ldots, x_{N}$ such that

$$
B_{1}^{0} \subset \bigcup_{i=1}^{N}\left(x_{i}+\varepsilon D\right) .
$$

Thus for every projection $P$ of rank $m$ we get

$$
\left|P B_{1}^{0}\right| \leq N \varepsilon^{m}|P D|,
$$

which means by (4)

$$
v_{m}\left(B_{1}^{0}\right) \leq\left(6^{n} \frac{\left|B_{1}^{0}\right|}{\left|B_{2}^{0}\right|}\right)^{1 / m} e_{n}\left(B_{2}^{0}, D\right) .
$$

Using Sudakov's inequality (see e.g., Theorem 5.5 of [26]) and (3), (5) we obtain

$$
\begin{aligned}
\frac{\ell\left(P_{E} B_{1}\right)}{\ell\left(B_{2}\right)} & \leq C(\lambda) \frac{1}{\sqrt{n} e_{n}\left(B_{2}^{0}, D\right)} \sum_{m=[c k]}^{n} \frac{\left(6^{n}\left|B_{1}^{0}\right| /\left|B_{2}^{0}\right|\right)^{1 / m} e_{n}\left(B_{2}^{0}, D\right)}{\sqrt{m}} \\
& \leq 4 C(\lambda)\left(6^{n}\left|B_{1}^{0}\right| /\left|B_{2}^{0}\right|\right)^{1 / c k}
\end{aligned}
$$

The result follows from the Santaló and the inverse Santaló inequalities.

Corollary 4.7 Let $K \subset \mathbb{R}^{n}$ be a convex body and let $a \in \mathbb{R}^{n}$ be an isomorphic Santaló point for $K$ (with constant $C^{\prime}$ ). Let $K_{1}=K-a$. For every $0<\lambda<1$, a random subspace $E \subset \mathbb{R}^{n}$ with $\operatorname{dim} E=[\lambda n]$, satisfies

$$
M\left(P_{E}\left(K_{1} \cap-K_{1}\right)\right) \leq C(\lambda) M\left(K_{1}-K_{1}\right),
$$

where $C(\lambda)$ depends on $C^{\prime}$ and $\lambda$.

Proof. By Proposition 4.6 it is sufficient to show that for some absolute constant $C$ we have

$$
\left|K_{1}-K_{1}\right| \leq C^{n}\left|K_{1} \cap-K_{1}\right| .
$$

This follows from the Rogers-Sheppard inequality, the definition of an isomorphic Santaló point and the symmetric inverse Santaló inequality. 
Remark 4.8 A global form of (6) says that there exists a unitary operator $U$ such that $M(B+U B) \leq C M\left(K_{1}-K_{1}\right)$, where $B=K_{1} \cap-K_{1}$ and $C$ is an absolute constant.

Remark 4.9 Inequality (7) was obtained in [18], and in [28].

Proof of Theorem 4.1. We start with general remarks on positions of a given convex body $K \subset \mathbb{R}^{n}$. Fix an arbitrary image $K_{2}=u(K)$ of $K$ under an operator $u$. Let $z$ be the Santaló point of $K_{2}$ and set $K_{3}=K_{2}-z$, so that 0 is the Santaló point for $K_{3}$. Pick $b \in \operatorname{Int} K_{3}$ such that $d_{K}=d_{K_{3}}=d_{K_{3}, b}$ and set $a=b / 2$. By Lemma 4.4, the polar $K_{3}^{a}$ with respect to $a$ satisfies $\left(\left|K_{3}\right|\left|K_{3}^{a}\right|\right)^{1 / n} \leq 2|D|^{2 / n}$. By Lemma $4.5, d_{K_{3}, a} \leq 3 d_{K}$. Now let $K_{1}=K_{3}-a$. Then $d_{0}\left(K_{1}, D\right)=d_{K_{3}, a} \leq 3 d_{K}$ and $K_{1}$ is clearly a position of $K$. Moreover, $K_{1}-K_{1}=u(K-K)$.

As recalled in the introduction, for the centrally symmetric convex body $K-K$ there exists a linear operator $u$ such that the body $B=u(K-K)$ satisfies $M(B) M\left(B^{0}\right) \leq C \kappa(B)=C \kappa(K-K)$. Let $K_{2}=u(K)$. Now, let $K_{1}$ be a position of $K$ constructed above, starting with $K_{2}$.

Since $B=K_{1}-K_{1}$, the first inequality follows immediately by Corollary 4.7 and (1). Since the Banach-Mazur distance satisfies $d(K-K, D) \leq$ $d_{0}\left(K_{1}, D\right) \leq 3 d_{K}$, the second estimate follows.

\section{Distances between Random Projections of Convex Bodies}

A natural way to measure a "non-symmetry" of convex bodies in $\mathbb{R}^{n}$ can be defined as the distance from a convex body $K \subset \mathbb{R}^{n}$ to the set of all symmetric bodies, which we shall denote by $\partial(K)$. That is, $\partial(K)=\inf d(K, B)$, where the infimum is taken over all symmetric convex bodies $B \subset \mathbb{R}^{n}$ (see [9] for different ways to measure the "non-symmetry"). By compactness, there exist $a \in K$ and a symmetric convex body $B \subset \mathbb{R}^{n}$ such that $\partial(K)=\widetilde{d}(K-a, B)$. Observe that we also have

$$
\begin{aligned}
\partial(K) & =\widetilde{d}(K-a,(K-a) \cap-(K-a)) \\
& =\widetilde{d}(K-a, \operatorname{conv}\{(K-a) \cup-(K-a)\}) .
\end{aligned}
$$

That is, $(K-a) \cap-(K-a)$ and conv $\{(K-a) \cup-(K-a)\}$ are two symmetric bodies closest to $K$. Also note, that for any $a \in K$

$$
\frac{1}{2}(K-K) \subset \operatorname{conv}\{(K-a) \cup-(K-a)\} \subset K-K .
$$

By John's theorem, $\partial(K) \leq d(K, D) \leq n$ for all $K \subset \mathbb{R}^{n}$. If $S$ is a regular simplex in $\mathbb{R}^{n}$, it is well known and easy to see that $d(S, D)=\partial(S)=n$. So the simplex is as far as possible from being symmetric and as far as possible 
from the Euclidean ball. Let us also mention that the simplex is the only body with the distance $n$ to the Euclidean ball ([25]). On the other hand, it was observed in [8] that $S$ has a $k=[(n+1) / 2]$-dimensional projection which is symmetric (in fact, it is isometric to the unit ball of $\ell_{1}^{k}$ ). In contrast, as our results show, orthogonal projections of $S$ on "random" $k$-dimensional subspaces are still very far from being symmetric.

Theorem 5.1 Let $S$ be a regular simplex in $\mathbb{R}^{n}$ having 0 as the center of mass, and let $p>1$. There exist constants $c>0$ and $C>0$ such that for $k \geq C \cdot p \cdot \log n$, the set of subspaces $E \in G_{n, k}$ such that projections $P=P_{E}$ satisfy

$$
c \sqrt{\frac{k}{p \cdot \log n}} \leq \partial(P S),
$$

has measure larger than $1-n^{-p}$.

Denote

$$
A=\sqrt{\frac{n}{k}} \int_{S^{n-1}}\left(\sum_{i=1}^{k} x_{i}^{2}\right)^{1 / 2} d \nu(x)=\frac{\sqrt{n} \Gamma\left(\frac{k+1}{2}\right) \Gamma\left(\frac{n}{2}\right)}{\sqrt{k} \Gamma\left(\frac{k}{2}\right) \Gamma\left(\frac{n+1}{2}\right)},
$$

where $\nu$ is the normalized rotationally invariant measure on the Euclidean sphere $S^{n-1}$ and $\Gamma(\cdot)$ is the Gamma function. Then $A=A(n, k)<1$ and $A \longrightarrow 1$ as $n, k \longrightarrow \infty$. We need a lemma, which follows from usual concentration inequalities on the sphere (we use it in the formulation from [11]).

Lemma 5.2 There exist two constants $c_{1}>0$ and $c>0$ such that for any $N$ and vectors $y_{1}, \ldots, y_{N} \in S^{n-1}$, any $\varepsilon>0$, and any integer $0<k<n$, the set of subspaces $E \in G_{n, k}$ such that projections $P=P_{E}$ satisfy

$$
\forall j:|| P y_{j}|-A \sqrt{k / n}| \leq A \varepsilon \sqrt{k / n},
$$

has measure larger than $1-c_{1} N \exp \left(-c \varepsilon^{2} k\right)$.

Proof of Theorem 5.1. Fix an arbitrary subspace $E \in G_{n, k}$. Using (8) it is easy to check that

$$
\begin{aligned}
\partial(P S) & =\inf _{a \in E} \widetilde{d}(P S-a,(P S-a) \cap-(P S-a)) \\
& =\inf _{a \in \mathbb{R}^{n}} \widetilde{d}(P(S-a), P(S-a) \cap-P(S-a)) .
\end{aligned}
$$

We shall estimate from below the expression under the latter infimum, for every $a \in \mathbb{R}^{n}$.

Assume first that $a=0$. Denote the vertices of the simplex by $v_{i}$, with $\left|v_{i}\right|=1$, for $1 \leq i \leq n+1$. Then $\sum v_{i}=0$ and $\left\langle v_{i}, v_{j}\right\rangle=-1 / n$ for $i \neq j$. 
Applying Lemma 5.2 for the set $\left\{v_{i}-v_{j}\right\}_{i, j=0}^{n+1}$, where $v_{0}=0$, we obtain that with probability larger than $1-c_{1}(n+2)^{2} \exp \left(-c \varepsilon^{2} k\right)$

$$
A(1-\varepsilon) \sqrt{k / n}\left|v_{i}-v_{j}\right| \leq\left|P v_{i}-P v_{j}\right| \leq A(1+\varepsilon) \sqrt{k / n}\left|v_{i}-v_{j}\right|
$$

for every $0 \leq i, j \leq n+1$ and some absolute constants $c_{1}, c>0$.

Since for every $x$ and $y$ one has $2\langle x, y\rangle=|x|^{2}+|y|^{2}-|x-y|^{2}$, then $\left|v_{i}-v_{j}\right|^{2}=2+2 / n$ for $i \neq j$, and so

$$
\left|\left\langle P v_{i}, P v_{j}\right\rangle\right| \leq A^{2} \frac{k}{n}\left(4 \varepsilon+(1+\varepsilon)^{2} / n\right) \leq A^{2} \frac{k}{n} 8 \varepsilon,
$$

for $\varepsilon \in(1 / n, 1)$.

Since $P S=\operatorname{conv}\left\{P v_{1}, \ldots, P v_{n+1}\right\}$ and $\sum P v_{i}=0$, to calculate the norm $\left\|-P v_{n+1}\right\|_{P S}$ we clearly need to use only vectors $P v_{i}$ for $1 \leq i \leq n$, that is,

$$
\left\|-P v_{n+1}\right\|_{P S}=\inf \left\{\sum_{i=1}^{n} \lambda_{i} \mid-P v_{n+1}=\sum_{i=1}^{n} \lambda_{i} P v_{i}, \lambda_{i} \geq 0\right\} .
$$

However, if $-P v_{n+1}=\sum_{i=1}^{n} \lambda_{i} P v_{i}$ for $\lambda_{i} \geq 0$ then

$$
A^{2}(1-\varepsilon)^{2} \frac{k}{n} \leq\left|\left\langle P v_{n+1}, P v_{n+1}\right\rangle\right| \leq \sum_{i=1}^{n} \lambda_{i}\left|\left\langle P v_{n+1}, P v_{i}\right\rangle\right| \leq A^{2} \frac{k}{n} 8 \varepsilon \sum_{i=1}^{n} \lambda_{i} .
$$

Thus we obtain $\left\|-P v_{n+1}\right\|_{P S} \geq(1-\varepsilon)^{2} /(8 \varepsilon)$ with probability larger than $1-\exp \left(\ln \left(c_{1}(n+2)^{2}\right)-\varepsilon^{2} c k\right)$. The choice

$$
\varepsilon=\sqrt{\frac{2 p \cdot \ln \left(c_{1}(n+2)^{2}\right)}{c k}}
$$

implies an estimate analogous to (9) for $n$ large enough (with a different absolute constant $c$ ). For small $n$ the estimate is trivial.

Now let $a \neq 0$. Of course, it is enough to consider the case $a \in S$ only. Arrange vertices of the simplex in such a way that $a \in \operatorname{conv}\left\{0, v_{1}, \ldots, v_{n}\right\}$. Then an obvious modification of the previous argument gives the same estimate as for $a=0$. This completes the proof.

The estimate in Theorem 5.1 is optimal, up to a logarithmic factor. This is a consequence of the following general upper estimate.

Theorem 5.3 Let $K \subset \mathbb{R}^{n}$ be a convex body such that $D$ is the ellipsoid of minimal volume for $K$. There exist constants $c>0$ and $C>0$ such that for $k \geq c\left(M_{K}^{*}\right)^{2} n$, the set of subspaces $E \in G_{n, k}$ such that projections $P=P_{E}$ satisfy

$$
d(P K, P D) \leq \widetilde{A} \sqrt{k \log n},
$$

where $\widetilde{A} \leq C \sqrt{n /(n-k)}$, has measure larger than $1-\exp (-c k)-\exp (-c(n-$ $k)$ ). 
By Dvoretzky's theorem, for $k \leq c\left(M_{K}^{*}\right)^{2} n$ an analogous distance is bounded by an absolute constant.

It should be noted that Theorem 5.3 is closely related to an isomorphic version of Dvoretzky's theorem recently proved by Milman and Schechtman ([20], [21]) for normed spaces, and later by Gordon, Guédon and Meyer ([8]) for arbitrary convex bodies (for $k \leq n / \log n$ ). The upper estimates proved in these papers are better than (11) by a logarithmic factor, which is slightly different in different ranges of $k$. However, except for the case of $k \leq n / \log n$ $([21],[8])$, the proofs do not give randomness.

Before we proceed with the proof of Theorem 5.3, let us recall a convenient notation, which will be used in the rest of this section. Given a convex body $K \subset \mathbb{R}^{n}$, let $a_{K}$ and $b_{K}$ denote the smallest real numbers for which the inclusions hold,

$$
\left(1 / b_{K}\right) D \subset K \subset a_{K} D .
$$

In particular, $a_{K}=b_{K^{0}}$ and $b_{K}=a_{K^{0}}$.

Remark 5.4 In this notation Theorem 2.1 means that for every convex body $K \subset \mathbb{R}^{n}$, a projection $P$ on a random $k$-dimensional subspace satisfies $b_{P K} \leq$ $2 \sqrt{n /(n-k)} M_{K}$.

Theorem 5.3 follows from Theorem 2.1 (see the remark above) and the following two lemmas. The first lemma is well known. In the dual form, this is essentially an upper estimate in Dvoretzky's theorem (valid without restrictions that $\varepsilon>0$ must be sufficiently small) see e.g., [19] (Theorem 4.2 and its proof).

Lemma 5.5 Let $K \subset \mathbb{R}^{n}$ be a convex body. There exist absolute constants $c>0$ and $C>0$ such that for any $1 \leq k \leq n$, the set of subspaces $E \in G_{n, k}$ such that projections $P=P_{E}$ satisfy $P K \subset r P D$, has measure larger than $1-\exp (-c k)$; here $r=c a_{K} \sqrt{k / n}$ for $k \geq\left(M_{K}^{*} / a_{K}\right)^{2} n$ and $r=c M_{K}^{*}$ for $k \leq\left(M_{K}^{*} / a_{K}\right)^{2} n$.

The next lemma comes from [1].

Lemma 5.6 Let $K \subset D$ be a convex body such that $D$ is ellipsoid of minimal volume for $K$. Then

$$
M_{K} \leq C \sqrt{n \log n}
$$

where $C$ is an absolute constant.

It is worth to mention that Barthe ([2]) has recently shown that the maximal value of $M_{K}$, over all convex bodies $K$ for which $D$ is the ellipsoid of minimal volume, is attained for the regular simplex $S$. Recall that $M_{S}$ is approximately equal to $\sqrt{n \log n}$. 
Remark 5.7 If $K$ in $\mathbb{R}^{n}$ is a body such that 0 is the Santaló point of $K$ (or, equivalently, 0 is the baricenter of $K^{0}$ ) and $D$ is the minimal volume ellipsoid for $K$ with such choice of origin then, using recent results of Guédon ([10], Theorems 2.2 and 2.3), the logarithmic factor in (11) can be removed. Indeed, dual form of Theorem 3 in [12] says that a projection $P$ on a random $k$-dimensional subspace satisfies

$$
b_{P K} \leq c(n /(n-k))^{3 / 2} \widetilde{M}_{K^{0}} \text { where } \widetilde{M}_{L}=\frac{1}{|L|} \int_{L}|x| d x
$$

for a convex body $L$ (cf. the previous remark). Recently Guédon has shown that if 0 is baricenter of $L$ and $D$ is the ellipsoid of maximal volume for $L$ with respect to baricenter than $\widetilde{M}_{L}<\sqrt{n}$ ([10], Theorem 2.2). Applying this estimate he obtained (in dual formulation) that $b_{P K} \leq \widetilde{A}_{0} \sqrt{n}$ for $\widetilde{A}_{0}=$ $c(n /(n-k))^{3 / 2}$ ([10], Theorem 2.3). Combining the last estimate with Lemma 5.5 we get $d(P K, P D) \leq \widetilde{A}_{0} \sqrt{k}$ instead of (11).

The following theorem is a standard application of estimates from Section 4. In the symmetric case it was proved in [4]. It shows that random sections and random projections of convex bodies are much closer together then it would follow from general estimates, and the distances between them admit the same estimates as for centrally symmetric bodies.

Theorem 5.8 Let $K_{1}$ and $K_{2}$ be convex bodies in $\mathbb{R}^{n}$ such that 0 is an isomorphic Santalo point for each of them (with constant $\left.c_{1}\right)$, and that $M\left(K_{i}-\right.$ $\left.K_{i}\right) M^{*}\left(K_{i}-K_{i}\right) \leq c_{2} \kappa\left(K_{i}-K_{i}\right)$, for $i=1,2$. Let $0<\lambda<1$. Then for two random subspaces $E_{1}$ and $E_{2}$ with $\operatorname{dim} E_{1}=\operatorname{dim} E_{2}=[\lambda n]$ one has

$$
\begin{aligned}
d\left(P_{E_{1}} K_{1}, K_{2}^{0} \cap E_{2}\right) & \leq C(\lambda) \sqrt{n} \kappa\left(K_{1}^{\prime}\right) \kappa\left(K_{2}^{\prime}\right) \\
& \leq C(\lambda) \sqrt{n} \ln \left(1+d_{K_{1}^{\prime}}\right) \ln \left(1+d_{K_{2}^{\prime}}\right),
\end{aligned}
$$

where $K_{i}^{\prime}=K_{i}-K_{i}$, for $i=1,2$, and $C(\lambda)$ depends on $c_{1}, c_{2}$ and $\lambda$.

Proof. We shall use the estimate that follows from Chevet's inequality ([3], in the symmetric case, see also [30]; and a general case follows by a standard modification). If $K$ and $L$ are convex bodies in $\mathbb{R}^{n}$, then

$$
d\left(K, L^{0}\right) \leq c\left(a_{K} M_{L}^{*}+a_{L} M_{K}^{*}\right)\left(b_{K} M_{L}+b_{L} M_{K}\right),
$$

where $c$ is an absolute constant.

By the proof of Theorem 4.1, for two random subspaces $F_{1}$ and $F_{2}$ of dimension $[\sqrt{\lambda} n]$ we have

$$
M\left(P_{F_{i}} K_{i}\right) M\left(K_{i}^{0}\right) \leq C(\lambda) \kappa\left(K_{i}-K_{i}\right),
$$

for $i=1,2$, where $C(\lambda)$ depends on $c_{1}, c_{2}$ and $\lambda$. 
By Theorem 2.1, for random subspaces $E_{i} \subset F_{i}$ of dimension $[\lambda n], i=1,2$, we have

$$
b_{P_{i} K_{i}} \leq 3(1-\sqrt{\lambda})^{-1 / 2} M\left(P_{i} K_{i}\right),
$$

where $P_{i}=P_{E_{i}}$.

Since $a_{P_{i} K_{i}} \leq c^{\prime} \sqrt{k} M^{*}\left(P_{i} K_{i}\right)$ (where $c^{\prime}$ is an absolute constant), then (12) implies

$$
\begin{aligned}
& d\left(P_{1} K_{1},\left(P_{2} K_{2}\right)^{0}\right) \\
& \leq c(1-\sqrt{\lambda})^{-1 / 2} \sqrt{k} M^{*}\left(P_{1} K_{1}\right) M^{*}\left(P_{2} K_{2}\right) M\left(P_{F_{1}} K_{1}\right) M\left(P_{F_{2}} K_{2}\right) \\
& \leq C^{\prime}(\lambda) \sqrt{n} \kappa\left(K_{1}-K_{1}\right) \kappa\left(K_{2}-K_{2}\right),
\end{aligned}
$$

where $C^{\prime}(\lambda)$ depends on $c_{1}, c_{2}$ and $\lambda$. This concludes the proof.

\section{The Proportional Dvoretzky-Rogers Factorization}

Results of this section are completely analogous of the theorem by Bourgain and Szarek ([5], see also [29]) for centrally symmetric convex bodies.

Theorem 6.1 Let $K \subset \mathbb{R}^{n}$ be a convex body, such that $D$ is the ellipsoid of minimal volume containing $K$. Let $\varepsilon \in(0,1)$ and set $k=[(1-\varepsilon) n]$. There exist vectors $x_{1}, x_{2}, \ldots, x_{k}$ in $K$, and an orthogonal projection $P$ in $\mathbb{R}^{n}$ with rank $P \geq k$ such that for all scalars $t_{1}, \ldots, t_{k}$

$$
c \varepsilon^{3}\left(\sum_{j=1}^{k}\left|t_{j}\right|^{2}\right)^{1 / 2} \leq\left\|\sum_{j=1}^{k} t_{j} P x_{j}\right\|_{P K} \leq \frac{6}{\varepsilon} \sum_{j=1}^{k}\left|t_{j}\right|,
$$

where $c>0$ is a universal constant.

Remark 6.2 For a centrally symmetric body $K$, the conclusion of the theorem can be equivalently expressed in terms of a factorization of the identity operator $i_{1,2}^{k}: \ell_{1}^{k} \rightarrow \ell_{2}^{k}$ through $K$. This is no longer true for non-symmetric bodies: the inequalities above imply the factorization of $i_{1,2}^{k}$ through $P K$ only.

In particular we have two immediate corollaries.

Corollary 6.3 Let $K \subset \mathbb{R}^{n}$ be a convex body such that $D$ is the ellipsoid of minimal volume containing $K$. For every $\varepsilon \in(0,1)$ there exist an orthogonal projection $P$ in $\mathbb{R}^{n}$ with $k=\operatorname{rank} P \geq(1-\varepsilon) n$ and an ellipsoid $\mathcal{D}$ on $P\left(\mathbb{R}^{n}\right)$ such that $(\varepsilon / 6) \mathcal{B}_{1}^{k} \subset P K \subset\left(C / \varepsilon^{3}\right) \mathcal{D}$, where $\mathcal{B}_{1}^{k}$ is the $l_{1}^{k}$-ball corresponding to $\mathcal{D}$. By duality, there exists a subspace $E \subset \mathbb{R}^{n}$ with $\operatorname{dim} E \geq k$ and an ellipsoid $\mathcal{D}$ on $E$ such that $c \varepsilon^{3} \mathcal{D} \subset K \cap E \subset(6 / \varepsilon) \mathcal{B}_{\infty}^{k}$, where $\mathcal{B}_{\infty}^{k}$ is the $l_{\infty}^{k}$-ball corresponding to $\mathcal{D}$. 
Corollary 6.4 Let $K \subset \mathbb{R}^{n}$ be a convex body such that $D$ is the ellipsoid of minimal volume containing $K$. For $\varepsilon \in(0,1)$, let $k=[(1-\varepsilon) n]$. Then there exist an orthogonal projection $P$ with $\operatorname{rank} P=k$ and a subspace $E \subset \mathbb{R}^{n}$ with $\operatorname{dim} E=k$ such that the Banach-Mazur distance satisfies

$$
\max \left(d\left(P K, B_{1}^{k}\right), d\left(K \cap E, B_{\infty}^{k}\right)\right) \leq\left(C / \varepsilon^{4}\right) \sqrt{n},
$$

where $C$ is an absolute constant and $B_{q}^{k}$ denotes the unit ball of $\ell_{q}^{k}$.

Remark 6.5 The interest in the second corollary is that the estimates of the distance of certain sections and projections of a (non-symmetric) convex body to the cube and the octahedron are of the same order in $n$ as for symmetric bodies; and they are better than general estimates.

Remark 6.6 The second part of Corollary 6.3 immediately shows that the proofs from [20] and [21] work in the non-centrally symmetric case as well. Thus we have the following theorem.

Theorem 6.7 For every $\log n \leq k \leq n / 2$, for every convex body $K$ in $\mathbb{R}^{n}$ there is a $k$-dimensional subspace $E$ of $\mathbb{R}^{n}$ such that $d_{E \cap K} \leq C \sqrt{k / \log (1+n / k)}$ with an absolute constant $C$.

This result in the non-centrally symmetric case was shown to be valid for $k \leq C n /(\log n)^{2}$ in [8] by a different method.

The proof of Theorem 6.1 essentially follows the argument from Szarek and Talagrand's paper [29], which however needs to be modified into a nonsymmetric setting.

Lemma 6.8 Let $K \subset \mathbb{R}^{n}$ be a compact convex body such that $D$ is the ellipsoid of minimal volume containing $K$. Let $\varepsilon \in(0,1)$. There exist $k \geq(1-\varepsilon) n$ and contact points $x_{1}, x_{2}, \ldots, x_{k}$ of $K$ and $D$ such that

$$
\operatorname{dist}\left(x_{j}, \operatorname{span}\left\{x_{i} \mid i \neq j, 1 \leq i \leq k\right\}\right) \geq \sqrt{\varepsilon},
$$

for $j=1, \ldots, k$.

Sketch of the proof. For sake of future reference we outline the known proof. By John's theorem there exist contact points $x_{1}, \ldots, x_{m}$ of $K$ and $D$ and positive scalars $c_{1}, \ldots, c_{m}$ which give a resolution of the identity, $x=\sum_{i} c_{i}\left(x_{i}, x\right) x_{i}$ for all $x \in \mathbb{R}^{n}$ and $\sum_{i} c_{i}=n$ (additionally, $\sum_{i} c_{i} x_{i}=0$, but we shall not use this). Then for any orthogonal projection $Q$ with $\operatorname{rank} Q=\ell$ one has $\max _{1 \leq i \leq m}\left|Q x_{i}\right| \geq(\ell / n)^{1 / 2}$. Indeed, $\ell=\operatorname{tr} Q=\sum_{i} c_{i}\left(x_{i}, Q x_{i}\right)=\sum_{i} c_{i}\left|Q x_{i}\right|^{2}$ $\leq \max _{i}\left|Q x_{i}\right|^{2} \sum_{i} c_{i}=n \max _{i}\left|Q x_{i}\right|^{2}$. 
Let $k=[(1-\varepsilon) n]+1$. For every subset $\sigma \subset\{1, \ldots, m\}$ with $|\sigma|=k$, consider $B_{\sigma}=\operatorname{conv}\left\{ \pm x_{i}: i \in \sigma\right\}$ and pick a subset $\sigma_{0}$ for which the $k$ dimensional volume of $B_{\sigma_{0}}$ is maximal. Then for all $j \in \sigma_{0}$ one has

$$
\begin{aligned}
& \operatorname{dist}\left(x_{j}, \text { span }\left\{x_{i} \mid i \neq j, i \in \sigma_{0}\right\}\right) \\
& =\left|Q x_{j}\right|=\max \left\{\left|Q x_{i}\right| \mid i \notin \sigma_{0} \text { or } i=j\right\} \geq \sqrt{\varepsilon},
\end{aligned}
$$

where $Q$ is the orthogonal projection with $\operatorname{ker} Q=\operatorname{span}\left\{x_{i} \mid i \neq j, i \in \sigma_{0}\right\}$.

Lemma 6.9 Let $\delta \in(0,1)$ and $\gamma>0$. Let $x_{1}, \ldots, x_{n}$ in $\mathbb{R}^{n}$ satisfy

$$
\operatorname{dist}\left(x_{j}, \operatorname{span}\left\{x_{i} \mid i \neq j\right\}\right) \geq \gamma,
$$

for $j=1, \ldots, n$. Then there exists a subset $\sigma \subset\{1, \ldots, n\}$ with $|\sigma| \geq(1-\delta) n$ and such that for all scalars $t_{1}, \ldots, t_{n}$

$$
\left|\sum_{j \in \sigma} t_{j} x_{j}\right| \geq c \gamma \delta\left(\sum_{j \in \sigma}\left|t_{j}\right|^{2}\right)^{1 / 2},
$$

where $c>0$ is an absolute constant.

This was proved in [29], as a combination of Proposition 4 and the argument from the proof of Corollary 5 . The dependence of the right hand side on $\gamma$ and $\delta$ was shown in the Proposition and Theorem 2 of [6].

The final lemma is taken from [5] (Lemma C), and for the sake of completeness we provide a short proof.

Lemma 6.10 Let $x_{1}, \ldots, x_{m} \in \mathbb{R}^{n}$ satisfy $\left|\sum t_{j} x_{j}\right| \geq\left(\sum\left|t_{j}\right|^{2}\right)^{1 / 2}$ for all scalars $\left(t_{j}\right)$. Let $\alpha \in(0,1)$ and let $P$ be an orthogonal projection in $\mathbb{R}^{n}$ with corank $P \leq \alpha m$. Then for every $\alpha<\delta<1$ there exists a subset $\sigma \subset\{1, \ldots, m\}$, with $|\sigma| \geq(1-\delta) m$ such that for all scalars $\left(t_{i}\right)$,

$$
\left|\sum_{j \in \sigma} t_{j} P x_{j}\right| \geq c(\delta-\alpha)^{3 / 2}\left(\sum_{j \in \sigma}\left|t_{j}\right|^{2}\right)^{1 / 2},
$$

where $c>0$ is an absolute constant.

Sketch of the proof. Similarly as in [5], without loss of generality we may assume that $n=m$ and the vectors $\left(x_{i}\right)$ are the standard unit vectors $\left(e_{i}\right)$ in $\mathbb{R}^{m}$. Thus we obtain the resolution of the identity in the space $E=P\left(\mathbb{R}^{m}\right)$, $x=\sum\left(P e_{i}, x\right) P e_{i}$ for $x \in E$. We have $k=\operatorname{dim} E \geq(1-\alpha) m$. Let $\delta^{\prime}=(\alpha+\delta) / 2$. By the proof of Lemma 6.8, there exists a subset $\sigma^{\prime}$ with $\ell=\left|\sigma^{\prime}\right| \geq k-\left(\delta^{\prime}-\alpha\right) k \geq\left(1-\delta^{\prime}\right) m$ such that for all $j \in \sigma^{\prime}$ we have $\operatorname{dist}\left(P e_{j}, \operatorname{span}\left\{P e_{i} \mid i \neq j, i \in \sigma^{\prime}\right\}\right) \geq \sqrt{\delta^{\prime}-\alpha}$. 
Therefore by Lemma 6.9 , there exists a subset $\sigma \subset \sigma^{\prime}$, with $|\sigma| \geq \ell-(\delta-$ $\left.\delta^{\prime}\right) \ell \geq(1-\delta) m$ such that

$$
\left|\sum_{j \in \sigma} t_{j} P e_{j}\right| \geq c\left(\delta^{\prime}-\alpha\right)^{1 / 2}\left(\delta-\delta^{\prime}\right)\left(\sum_{j \in \sigma}\left|t_{j}\right|^{2}\right)^{1 / 2},
$$

which immediately concludes the proof.

Remark 6.11 The conclusion of Lemma 6.10 holds as well if the assumption on the vectors $\left(x_{i}\right)$ is replaced by the condition that the vectors give the John's resolution of the identity: Let $m \geq n$ and let $x_{i} \in \mathbb{R}^{n}$, with $\left|x_{i}\right|=1$, and $c_{i}>0$, for $i=1, \ldots, m$ satisfy $x=\sum_{i} c_{i}\left(x_{i}, x\right) x_{i}$ for all $x \in \mathbb{R}^{n}$ and $\sum_{i} c_{i}=n$. This follows from a similar proof as above, preceded with a slightly modified argument as in the proof of Lemma 6.8.

Proof of Theorem 6.1. Let $D$ be the ellipsoid of minimal volume for $K$. By Lemma 6.8 there exist $n \geq k \geq(1-\varepsilon / 3) n$ and contact points $x_{1}, \ldots, x_{k}$ of $K$ and $D$ that satisfy the conclusion of the lemma with the lower estimate $\sqrt{\varepsilon / 3}$. Thus, by Lemma 6.9 , there exists a subset $\sigma^{\prime} \subset\{1, \ldots, k\}$ with $m=$ $\left|\sigma^{\prime}\right| \geq k-(\varepsilon / 3) k \geq(1-2 \varepsilon / 3) n$ that the lower $\ell_{2}$-estimate of the lemma is satisfied with the function $c^{\prime} \varepsilon^{3 / 2}$, where $c^{\prime}>0$ is an absolute constant.

It is not difficult to construct, for an arbitrary $\beta \in(0,1)$, an orthogonal projection $P$ with corank $P=[\beta m]$ such that $-P x_{j} \in \beta^{-1} P K$ for every $j \in \sigma^{\prime}$. Indeed, partition the set $\sigma^{\prime}$ into $[\beta m]$ disjoint subsets $A_{s}$, with $\left|A_{s}\right| \leq$ $[1 / \beta]+1$ for all $s$. Let $z_{s}=\sum_{i \in A_{s}} x_{i}$, and let $P$ be the orthogonal projection with ker $P=\operatorname{span}\left\{z_{s}\right\}$. Since $P z_{s}=0$, then $-P x_{j} \in[1 / \beta] \operatorname{conv}\left\{P x_{i}: i \neq\right.$ $\left.j, i \in A_{s}\right\}$, for all $j \in A_{s}$ and all $s$. Thus $P$ is an orthogonal projection of corank $[\beta m]$ such that $-P x_{j} \in[1 / \beta] P K$, for all $j \in \sigma^{\prime}$.

Let $\beta=\varepsilon / 6$ and let $P$ be the corresponding orthogonal projection. Then, by Lemma 6.10 , with $\alpha=\varepsilon / 6$ and $\delta=\varepsilon / 3$ we get a set $\sigma \subset \sigma^{\prime},|\sigma| \geq$ $m-(\varepsilon / 3) m \geq(1-\varepsilon) n$ such that for all scalars $\left(t_{i}\right)$,

$$
\left|\sum_{j \in \sigma} t_{j} P x_{j}\right| \geq c \varepsilon^{3}\left(\sum_{j \in \sigma}\left|t_{j}\right|^{2}\right)^{1 / 2}
$$

where $c>0$ is a universal constant. Since $|\sigma| \geq k$, by relabelling vectors $x_{j}$ we get the left hand side of the required inequality:

$$
\left\|\sum_{j=1}^{k} t_{j} P x_{j}\right\|_{P K} \geq\left|\sum_{j=1}^{k} t_{j} Q x_{j}\right| \geq c \varepsilon^{3}\left(\sum_{j=1}^{k}\left|t_{j}\right|^{2}\right)^{1 / 2} .
$$

The right hand side is obvious from the triangle inequality, since letting $B=P K \cap(-P K)$ we get a convex centrally symmetric body in $P\left(\mathbb{R}^{n}\right)$, and $\left\|P x_{j}\right\|_{P K} \leq\left\|P x_{j}\right\|_{B} \leq 6 / \varepsilon$, for all $j \in \sigma$. 


\section{References}

1. Banaszczyk W., Litvak A.E., Pajor A., Szarek S.J. (1999) The flatness theorem for non-symmetric convex bodies via the local theory of Banach spaces. Math. Oper. Res. 24(3):728-750

2. Barthe F. (1998) An extremal property of the mean width of the simplex. Math. Ann. 310(4):685-693

3. Benyamini Y., Gordon Y. (1981) Random factorization of operators between Banach spaces. J. Anal. Math. 39:45-74

4. Bourgain J., Milman V.D. (1986) Distances between normed spaces, their subspaces and quotient spaces. Integral Equations Operator Theory 9:31-46

5. Bourgain J., Szarek S.J. (1988) The Banach-Mazur distance to the cube and the Dvoretzky-Rogers factorization. Israel J. Math. 62(2):169-180

6. Giannopoulos A.A. (1995) A note on the Banach-Mazur distance to the cube. In: Geometric Aspects of Functional Analysis (Israel, 1992-1994), 67-73, Oper. Theory Adv. Appl., 77, Birkhäuser, Basel

7. Gordon Y. (1988) On Milman's inequality and random subspaces which escape through a mesh in $\mathbb{R}^{n}$. In: Geometric Aspects of Functional Analysis (1986/87), 84-106, Lecture Notes in Math., 1317, Springer, Berlin-New York

8. Gordon Y., Guédon O., Meyer M. (1998) An isomorphic Dvoretzky's theorem for convex bodies. Studia Math. 127(2):191-200

9. Grünbaum B. (1963) Measures of symmetry for convex sets. Proc. Sympos. Pure Math., Vol. VII, 233-270. Amer. Math. Soc., Providence, R.I.

10. Guédon O. (1998) Sections euclidiennes des corps convexes et inégalités de concentration volumique. Thèse de doctorat de mathématiques, Université de Marne-la-Vallée

11. Johnson W.B., J. Lindenstrauss J. Extensions of Lipschitz mappings into a Hilbert space. In: Conference in Modern Analysis and Probability (New Haven, Conn., 1982), 189-206

12. Litvak A.E., Milman V.D., Pajor A. (1999) The covering numbers and "low $M^{*}$-estimate" for quasi-convex bodies. Proc. Amer. Math. Soc. 127:1499-1507

13. Mankiewicz P., Tomczak-Jaegermann N. (1990) Pathological properties and dichotomies for random quotients of finite-dimensional Banach spaces. In: Geometry of Banach Spaces (Strobl, 1989), 199-217, London Math. Soc. Lecture Note Ser., 158, Cambridge Univ. Press, Cambridge

14. Milman V.D. (1985) Almost Euclidean quotient spaces of subspaces of a finite dimensional normed space. Proc. Amer. Math. Soc. 94:445-449

15. Milman V.D. (1985) Random subspaces of proportional dimension of finite dimensional normed spaces: approach through the isoperimetric inequality. In: Banach Spaces (Columbia, Mo., 1984), 106-115, Lecture Notes in Math., 1166, Springer, Berlin-New York

16. Milman V.D. (1990) A note on a low $M^{*}$-estimate. In: Geometry of Banach Spaces (Strobl, 1989), 219-229, London Math. Soc. Lecture Note Ser., 158, Cambridge Univ. Press, Cambridge

17. Milman V.D. (1991) Some applications of duality relations. Geometric Aspects of Functional Analysis (1989-90), 13-40, Lecture Notes in Math., 1469, Springer, Berlin

18. Milman V.D., Pajor A. Entropy and asymptotic geometry of non-symmetric convex bodies. Advances in Math., to appear; see also (1999) Entropy methods 
in asymptotic convex geometry. C.R. Acad. Sci. Paris, S.I. Math., 329(4):303308

19. Milman V.D., Schechtman G. (1985) Asymptotic theory of finite-dimensional normed spaces. Lecture Notes in Math., 1200, Springer, Berlin-New York

20. Milman V.D., Schechtman G. (1995) An "isomorphic" version of Dvoretzky's theorem. C. R. Acad. Sci. Paris Sr. I Math. 321:541-544

21. Milman V.D., Schechtman G. (1998) An "isomorphic" version of Dvoretzky's theorem, II. Convex Geometry, MSRI Publications, Vol. 34

22. Pajor A., Tomczak-Jaegermann N. (1985) Remarques sur les nombres d'entropie d'un opérateur et de son transposé. C. R. Acad. Sci. Paris Sr. I Math. 301(15):743-746

23. Pajor A., Tomczak-Jaegermann N. (1986), Subspaces of small codimension of finite-dimensional Banach spaces. Proc. Amer. Math. Soc. 97(4):637-642

24. Pajor A., Tomczak-Jaegermann N. (1989) Volume ratio and other $s$-numbers of operators related to local properties of Banach spaces. J. Funct. Anal. $87(2): 273-293$

25. Palmon O. (1992) The only convex body with extremal distance from the ball is the simplex. Israel J. Math. 80(3):337-349

26. Pisier G. (1989) The Volume of Convex Bodies and Banach Space Geometry. Cambridge University Press, Cambridge

27. Rudelson M. Sections of the difference body. Discrete and Computational Geometry, to appear.

28. Rudelson M. Distances between non-symmetric convex bodies and the $M M^{*}$ estimate. Positivity, to appear.

29. Szarek S.J., Talagrand M. (1989) An "isomorphic" version of the Sauer-Shelah lemma and the Banach-Mazur distance to the cube. In: Geometric Aspects of Functional Analysis (1987-88), 105-112, Lecture Notes in Math., 1376, Springer, Berlin-New York

30. Tomczak-Jaegermann N. (1989) Banach-Mazur distances and finitedimensional operator ideals. Pitman Monographs and Surveys in Pure and Applied Mathematics, 38. Longman Scientific \& Technical, Harlow; John Wiley \& Sons, Inc., New York 